\title{
Optical Responses in Asymmetric Inverse Parabolic Quantum Wells: Effects of Laser Fields and Hydrostatic Pressure
}

\author{
M.E. Mora-RAmos ${ }^{a, b, c}$, A.L. Morales ${ }^{b}$ And C.A. DuquE ${ }^{b}$ \\ ${ }^{a}$ Facultad de Ciencias, Universidad Autónoma del Estado de Morelos, CP. 62209, México \\ ${ }^{b}$ Grupo de Materia Condensada-UdeA, Instituto de Física, Facultad de Ciencias Exactas y Naturales \\ Universidad de Antioquia UdeA, Calle 70 No. 52-21, Medellín, Colombia \\ ${ }^{c}$ Física Teórica y Aplicada, Escuela de Ingeniería de Antioquia, AA 7516, Medellín, Colombia
}

\begin{abstract}
We report the theoretical calculation of the electronic states in a $\mathrm{Al}_{x} \mathrm{Ga}_{1-x}$ As-based quantum well with inverse parabolic confinement under the combined effects of the intense laser field and hydrostatic pressure. Calculations are in the effective mass and parabolic band approximations and using a variational procedure and the so-called Floquet method in order to obtain the energies and wave functions for the conduction band states. We use the obtained information to investigate the intersubband-related nonlinear optical absorption and optical rectification coefficients.
\end{abstract}

DOI: $10.12693 /$ APhysPolA.125.202

PACS: $42.65 .-\mathrm{k}, 73.63 . \mathrm{Hs}, 73.21 .-\mathrm{b}, 74.25 . \mathrm{Gz}$

\section{Introduction}

Research on the optical properties in semiconductor heterostructures has been ongoing for more than two decades now [1-4]. The dressed atom approach was extended by Brandi et al. $[5,6]$ to treat the influence of the laser field upon a semiconductor system. On the other hand, the intense laser field (ILF) effects on the confining potential and electron states in quantum wells (QWs) were studied by Lima et al. [7]. They discovered a laser-induced transition from single to double potential well profile in the conduction band of this kind of low-dimensional systems. We have investigated the linear and nonlinear optical properties in rectangular quantum QWs under the effect of intense laser radiation and applied electromagnetic fields [8]. In addition, some of us have calculated the electron states and related nonlinear optical responses in asymmetric inverse parabolic QWs [9].

In this report we are presenting some results on the optical response of asymmetric inverse parabolic quantum wells in the presence of ILF and hydrostatic pressure. In Sect. 2 we give a brief outline of the theoretical model used, and in Sect. 3 the obtained results are discussed. Finally, the main conclusions of the work are stated in Sect. 4.

\section{Outlook of the theoretical model}

The system under study is a GaAs-based asymmetric single QW grown along the $z$-direction, with rectangular potential barriers and well region profile in the form of an inverse parabola. The configuration formed turns out to be that of a double asymmetric QW. The details of the calculation of single-particle band states within the effective mass approximation can be found in Ref. [9]. Once we identify the main energy transition $\left(E_{i} \rightarrow E_{j}\right)$ in the conduction band of the system, the corresponding electric dipole moment matrix element $M_{i j}=\left\langle\psi_{i}|z| \psi_{j}\right\rangle$ will be the only remaining quantity to evaluate. In addition, the study takes into account the influence of the hydrostatic pressure by introducing the corresponding dependences of the electron effective mass and the dielectric constant on $P: m_{\mathrm{e}} / m_{0}=0.0665+5.7076 \times 10^{-4} P$. The dimensions of the structure change according to the expression $L(P)=L(0)\left[1-\left(S_{11}+2 S_{12}\right) P\right]$. The dependence on the pressure of the intense laser field parameter is given by $\alpha_{0}(P)=\alpha_{0} m_{\mathrm{e}}(0) / m_{\mathrm{e}}(P)$. The confinement potential in absence of laser effects is $V=V_{0}$ for $|x|>L / 2$ and $V=V_{0}\left(1-4 / L^{2}\right)\left(x+x_{0}\right)^{2}$ for $|x| \leq L / 2$. The evaluation of the linear and nonlinear optical absorption, as well as the nonlinear optical rectification (NOR) coefficients will follow the corresponding approaches introduced in Refs. [10-12].

\section{Results and discussion}

Figures 1 and 2 show the potential profile and the electron probability densities (PDs) of the lowest two-electron confined states in an inverse parabolic QW. For illustration, the PDs are schematically positioned over the corresponding energy values. Here, $\alpha_{0}=0$ and $L=20 \mathrm{~nm}$. In Fig. 1a $\left(x_{0}=0\right)$, the black line corresponds to the even state and the red one to the odd state. They essentially coincide, given that the two wells are identical and uncoupled by the separating barrier. In the case depicted in Fig. 1b, the asymmetric parabolic configuration in the potential well region has shifted to the left. In this case, both states become essentially confined within the right-hand QW. The well appearing at the right behaves, in fact, as a small perturbation.

Reducing the value of the $x_{0}$ parameter, we can observe that the first excited state also extends - and mostly confines - over the left-hand QW region (Fig. 2a). With 

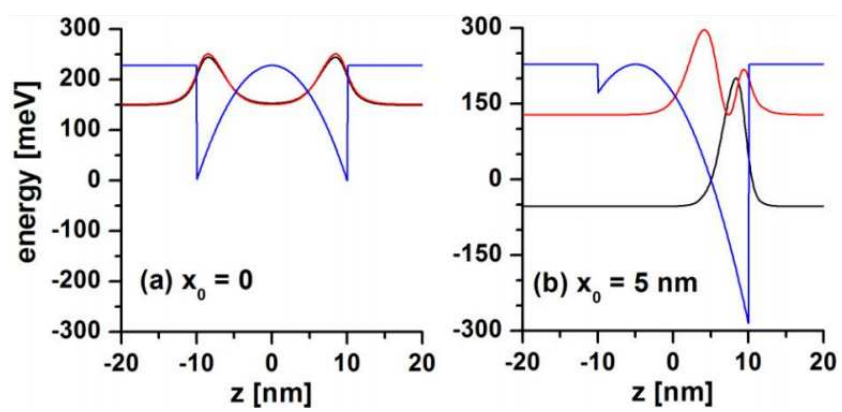

Fig. 1. Confining potential profile and the probability densities of the first two electron confined states in: (a) a symmetric inverse parabolic QW, and (b) an asymmetric inverse parabolic QW.
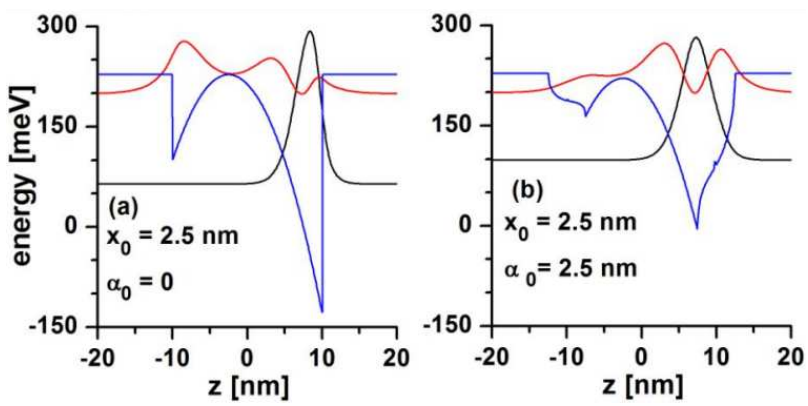

Fig. 2. Confining potential profile and the probability densities of the first two electron confined states in: (a) an asymmetric inverse parabolic QW; and (b) an asymmetric inverse parabolic QW with the intense laser field effect.

the same configuration, the inclusion of an ILF with $\alpha_{0}=$ $2.5 \mathrm{~nm}$ causes the modification of the confining potential profile in such a way that the well depths diminish and their effective widths augment (Fig. 2b). The increase in the effective width of the right-hand QW allows for the confinement of both states within it, although the
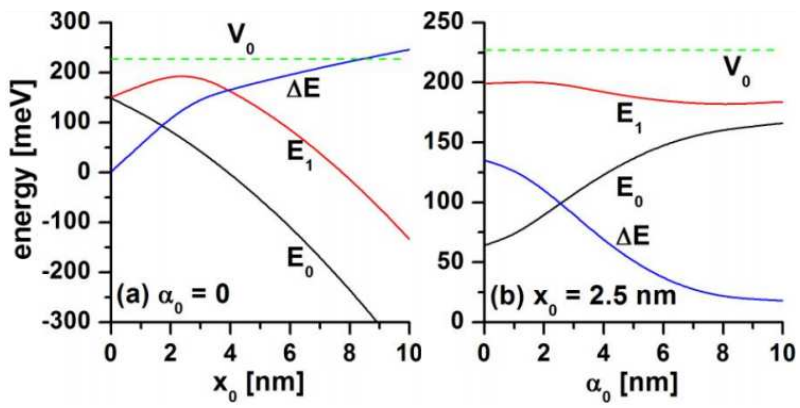

Fig. 3. Energies of the two lowest confined states and transition energy difference (blue line) in an inverse parabolic QW with $L=20 \mathrm{~nm}$. (a) As functions of the inverted parabola's vertex position and zero laser field. (b) As functions of the intense laser parameter for a fixed parabolic configuration. left-hand QW still supports a part of the second state probability density.

Figure 3 contains the calculated energies of the two lowest confined states in an inverse parabolic QW with the same geometrical configuration already discussed. In Fig. 3a, these energies are presented as functions of the parabola vertex position for zero laser field; whereas Fig. 3b depicts the energies as functions of the ILF parameter when the shape of the inverted parabola is fixed, $x_{0}=2.5 \mathrm{~nm}$. The dashed line at the top represents the maximum of the confining potential energy in the structure. The blue line is the corresponding evolution of the energy difference between the two states which, ultimately, tells us about the direction in which the resonant peaks of the optical responses move. The decreasing behavior of the energies in the case of zero laser field can be explained by observing Fig. 2a. Augmenting the value of $x_{0}$ makes the right hand QW to deepening and the two energy levels become more strongly confined. This effect is more pronounced for the ground state. For this reason, the transition energy difference shows a growing variation.

On the other hand, the increase in the ILF parameter for a fixed parabola shape leads to the rise of the QW bottom, which pushes upwards the energy position of the ground state, whilst the first excited one descends due to the laser-induced widening of the QW. Therefore, we are observing a monotonously decreasing variation of the transition energy difference.
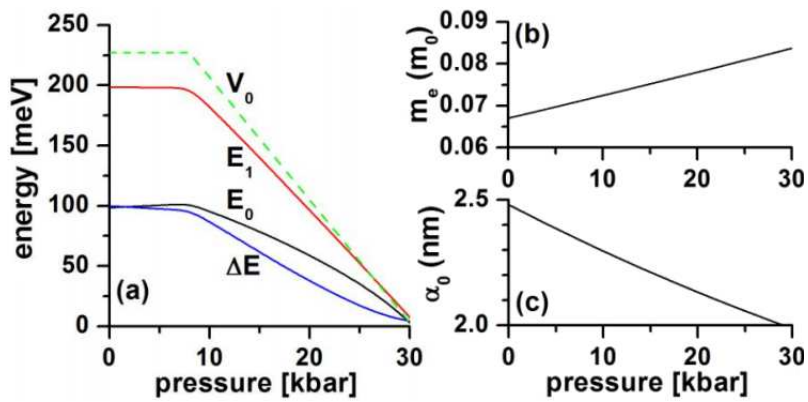

Fig. 4. (a) Energies of the two lowest confined states, transition energy difference and maximum of the confining potential as functions of the hydrostatic pressure in a GaAs-based inverse parabolic QW. Variations with pressure of the electron effective mass (b) and intense laser field parameter (c). Here, $\alpha_{0}=x_{0}=2.5 \mathrm{~nm}$, $L=20 \mathrm{~nm}$.

In Fig. 4 we are presenting the effects of the hydrostatic pressure applied over the system under study. The variations of the state energies, potential well maximum and transition energy difference appear in Fig. 4a as functions of the pressure for the configuration in which $\alpha_{0}=x_{0}=2.5 \mathrm{~nm}$ and $L=20 \mathrm{~nm}$. In addition, the main quantities entering the Schrödinger equation appear depicted as functions of the pressure in Fig. $4 \mathrm{~b}$. The behaviors of these quantities help to understand the variations of the energies in Fig. $4 \mathrm{a}$. 


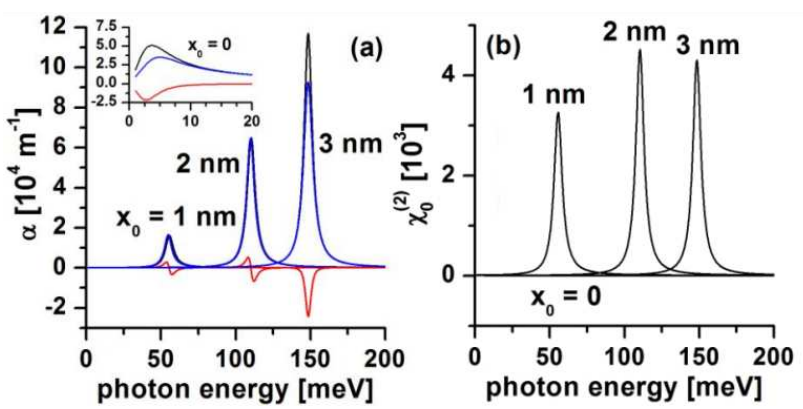

Fig. 5. (a) Linear (black), nonlinear (red) and total (blue) optical absorption coefficients. (b) NOR coefficient. Different values of $x_{0}$ are considered, for zero laser field. $L=20 \mathrm{~nm}$.

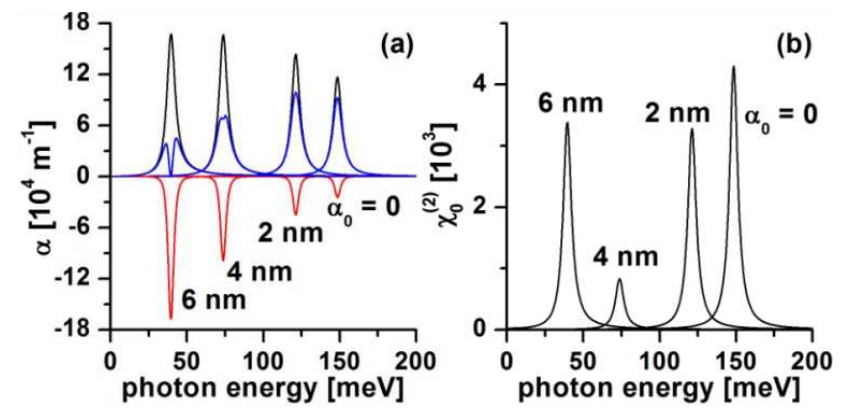

Fig. 6. (a) Linear (black), nonlinear (red) and total (blue) optical absorption coefficients. (b) NOR coefficient. Different values of $\alpha_{0}$ are considered, for $x_{0}=3 \mathrm{~nm}$ and $L=20 \mathrm{~nm}$.

Figures 5 and 6 show the coefficients of linear, third-order nonlinear and total absorption (at the left, Figs. 5a and $6 \mathrm{a}$ ) and that of the NOR (at the right, Figs. 5b and $6 \mathrm{~b}$ ); all as functions of the incident photon energy. In Fig. 5, the parabola vertex position enters as a parameter, whilst in Fig. 6 this is done for the laser-field one.

A blueshift is observed with the increment of the value of the parabola vertex position, which is compatible with the behavior of the transition energy difference shown in Fig. 3a. In the symmetric case, the NOR is zero because the dipole moment matrix element identically vanishes. On the other hand, augmenting the ILF parameter causes a redshift of the resonant peaks, consistent with the variation of the transition energy difference in Fig. 3b. It can be readily seen that the growth of each of these two input parameters $\alpha_{0}, x_{0}$, leads to a significant increment in the nonlinear contribution to the optical absorption coefficient for the same incident light intensity, here taken as $5 \times 10^{3} \mathrm{~W} / \mathrm{cm}^{2}$.

\section{Conclusions}

In this work, we have studied the electron energy states and the related linear and nonlinear optical absorption and nonlinear optical rectification in an inverse parabolic quantum well under the effects of the intense laser fields and hydrostatic pressure. In general, changing the symmetry of the inverted parabola in the well region may lead to the blueshift of the corresponding resonant peaks, and the increment in the laser field intensity causes a redshifts of these signals. The increase in these two input parameters enhances the nonlinear contribution to the optical absorption in the system under study.

\section{Acknowledgments}

M.E.M.R. acknowledges support from Mexican CONACYT through grant CB-2008-101777, and through sabbatical grant 2011-2012 No. 18036. He also thanks Universidad de Antioquia and Escuela de Ingeniería de Antioquia for hospitality during his sabbatical stay. This research was partially supported by Colombian Agencies: CODI-Universidad de Antioquia (Estrategia de Sostenibilidad 2013-2014 de la Universidad de Antioquia and the project: E01535-Efectos de la presión hidrostática y de los campos eléctrico y magnético sobre las propiedades ópticas no lineales de puntos, hilos y anillos cuánticos de GaAs- $(\mathrm{Ga}, \mathrm{Al}) \mathrm{As}$ y $\left.\mathrm{Si} / \mathrm{SiO}_{2}\right)$, Facultad de Ciencias Exactas y Naturales-Universidad de Antioquia (CAD-exclusive dedication project 2012-2013), and by El Patrimonio Autónomo Fondo Nacional de Financiamiento para la Ciencia, la Tecnología y La Innovacion, Francisco José de Caldas. This work was carried out with the help of CENAPAD-SP, Brazil.

\section{References}

[1] V. Mitin, V. Kochelap, M.A. Stroscio, Quantum Heterostructures, Cambridge University Press, Cambridge 1999.

[2] P. Harrison, Quantum Wells, Wires and Dots, 2nd ed., Wiley, Chichester 2005.

[3] E. Rosencher, Ph. Bois, Phys. Rev. B 44, 11315 (1991).

[4] D. Ahn, S.-L. Chuang, IEEE J. Quant. Electron. QE23, 2196 (1987).

[5] H.S. Brandi, A. Latgé, L.E. Oliveira, Phys. Status Solidi B 210, 671 (1998).

[6] H.S. Brandi, A. Latgé, L.E. Oliveira, Phys. Rev. B 70, 153303 (2004).

[7] F.M.S. Lima, M.A. Amato, O.A.C. Nunes, A.L.A. Fonseca, B.G. Enders, E.F. da Silva Jr., J. Appl. Phys. 105, 123111 (2009).

[8] M.E. Mora-Ramos, C.A. Duque, E. Kasapoglu, H. Sari, I. Sökmen, J. Lumin. 132, 901 (2012).

[9] C.A. Duque, M.E. Mora-Ramos, Superlatt. Microstruct. 54, 61 (2013).

[10] C.A. Duque, E. Kasapoglu, S. Şakiroglu, H. Sari, I. Sökmen, Appl. Surf. Sci. 257, 2313 (2011).

[11] I. Karabulut, M.E. Mora-Ramos, C.A. Duque, J. Lumin. 131, 1502 (2011).

[12] I. Karabulut, C.A. Duque, Physica E 43, 1405 (2011). 